\title{
Método para la formación de grupos colaborativos mediante disponibilidad léxica
}

\section{Method for the Formation of Collaborative Groups Using Lexical Availability}

Darío Fernando Rojas Díaz (*) dfrojas@gmail.com

Carolina Zambrano Matamala $\left(^{*}\right)$ carolinazambrano@gmail.com

Pedro Salcedo Lagos (*) paslagos@gmail.com

(*) Universidad de Concepción

(Recibido: 21 de julio de 2017; Aceptado para su publicación: 6 de octubre de 2017)

Cómo citar: Rojas, D. F., Zambrano, C. y Salcedo, P. (2019). Método para la formación de grupos colaborativos mediante disponibilidad léxica. Revista Electrónica de Investigación Educativa, 21, e36, 1-12. doi:10.24320/redie.2019.21.e36.2095

\section{Resumen}

La presente investigación propone un método de formación automática de grupos colaborativos que utiliza las pruebas de disponibilidad léxica para la selección de los miembros. El método es fundamentado mediante la comparación de lexicones y enfoques de análisis de disponibilidad léxica utilizando una muestra de 394 estudiantes. La evaluación del método propuesto se realizó a través de un estudio de caso con 18 estudiantes en una actividad colaborativa. Los resultados encontrados muestran que, en las actividades de mayor complejidad, los grupos conformados a través del método propuesto tardan un menor tiempo en responder y generan respuestas más sintéticas y, por lo tanto, de mejor calidad. De lo expuesto se puede concluir que es factible, mediante el método propuesto, conformar grupos colaborativos sobre la base de la cuantificación de los niveles de interacción y comunicación reflejados en las características de la disponibilidad léxica de sus integrantes.

Palabras clave: Aprendizaje colaborativo, disponibilidad léxica, conformación automática de grupos, proceso de interacción.

\section{Abstract}

A method of automatic formation of collaborative groups that uses the lexical availability tests to select the group members is proposed. The method is based on the comparison of lexicons and lexical availability analyses approaches using a sample of 394 students. The evaluation of the suggested method was carried out through a case study with 18 students in a collaborative activity. The results show that in higher-complex activities the groups assigned through the suggested method spend less time to answer and generate more synthetic responses and, therefore, of better quality. It can be concluded that it is feasible to make collaborative groups based on the quantification of the levels of interaction and communication reflected in the characteristics of the group members' lexical availability. 


\section{Introducción}

Desde la perspectiva educacional, la teoría sociocultural de Vigotsky afirma que las personas aprenden a través de las interacciones sociales y su cultura (Álvarez, 2010; Salas, 2001). Específicamente en el aula, hay estrategias de aprendizaje como las basadas en grupos colaborativos, donde la interacción entre los miembros puede ser un componente esencial para la adquisición de conocimientos, habilidades y actitudes (Ariza y Oliva, 2000; Carrió, 2007).

Con este propósito, la elección de los integrantes de un grupo puede ser crucial para fomentar la cantidad y calidad de las interacciones producidas en el proceso de colaboración, por lo que parece vital definir estrategias de conformación de grupos que tengan en cuenta las diferencias entre sus integrantes. Estas estrategias deben tener en cuenta el contexto donde se producen y los elementos comunicacionales de cada integrante, permitiendo agruparlos de tal forma que la heterogeneidad de sus características posibilite generar un ambiente de desarrollo, donde sus diferencias den lugar a la interacción y colaboración necesarias para alcanzar los objetivos comunes de aprendizaje. A partir esa necesidad, el objetivo de esta investigación es proponer un método de conformación automática de grupos colaborativos apoyado por software, que utiliza la disponibilidad léxica de los estudiantes como criterio para maximizar la heterogeneidad de los integrantes de cada grupo y la homogeneidad de los grupos entre sí. De esta forma se busca enriquecer el proceso de interacción entre los individuos para alcanzar un mayor nivel de aprendizaje individual.

Existe evidencia a favor de que los alumnos pueden afrontar mejor los problemas o necesidades derivadas de las relaciones interpersonales cuando trabajan en ambientes de aprendizaje colaborativos o cooperativos (Arias-Gundín, Fidalgo y García, 2008). En este contexto, el aprendizaje colaborativo y el aprendizaje cooperativo pueden ser considerados estrategias metodológicas de aprendizaje en grupo de amplia utilización, que están asociadas al desarrollo psicosocial de los integrantes. Por un lado, el aprendizaje colaborativo se apoya en la idea de que la interdependencia entre los individuos debe ser abordada por los mismos integrantes del grupo, requiriendo habilidades sociales más desarrolladas por parte de los alumnos y pasando a ser el rol del profesor el de un mediador cognitivo (Collazos y Mendoza, 2006; Galindo y Arango, 2009; Maldonado y Vásquez, 2008). Por otro lado, el aprendizaje cooperativo sugiere una dirección más estricta por parte del profesor, lo que puede implicar la asignación de roles y tareas específicas a los miembros (Lage, 2001).

En consecuencia, los enfoques de aprendizaje colaborativo y cooperativo pueden diferenciarse principalmente en el nivel de estructuración de la actividad que involucra (Zañartu, 2013). Esto influye en el nivel de interacción social producido entre los participantes, el cual puede ser menor en el enfoque cooperativo debido a la distribución de tareas entre los miembros y la consecuente posibilidad de definir objetivos de aprendizaje distintos para cada integrante, lo que hace menos necesaria la interacción entre los sujetos para la consecución de sus metas.

Por lo anterior, la presente investigación se enmarca en el aprendizaje colaborativo, ya que centra su interés en el nivel de interacción de colaboración producido entre los miembros de un grupo y la influencia que esta interacción tiene en la consecución de los objetivos de la tarea colaborativa, lo que direcciona a definir actividades que tengan bajo nivel de estructuración y distribución.

En estos ambientes colaborativos los individuos involucrados afectan mutuamente su aprendizaje, producto de la interacción social producida -la que denominaremos "interacción colaborativa", cuya meta es ayudar a alcanzar los objetivos comunes dentro de un proceso de aprendizaje colaborativo.

La interacción colaborativa se desarrolla fundamentalmente a través de los procesos comunicativos derivados del uso del lenguaje, permitiendo a cada miembro internalizar el aprendizaje e incorporarlo a su propio aparato cognoscitivo (Pérez, 2007). Así, el aprendizaje es un proceso cognitivo individual influenciado por los factores externos (Ariza y Oliva, 2000) que se producen por el intercambio verbal entre los miembros del grupo (Lucero, 2003). Este intercambio verbal puede ser visto como un proceso continuo de negociación de significados y de establecimiento de contextos mentales compartidos (Coll y 
Solé, 1990), que lleva a los participantes a internalizar conceptos consensuados que constituyen la base рага nuevos aprendizajes. De esta forma, la asimetría de conocimiento entre los miembros de un grupo puede conducir a un mayor nivel de interacción colaborativa (Zañartu, 2013) y, por lo tanto, a un aumento en la cantidad de negociaciones de significados mediante el intercambio verbal.

Desde el proceso de comunicación que subyace en ese intercambio verbal es posible medir el caudal léxico disponible por los participantes cuando se define un contexto y tema de trabajo. Este caudal denominado "disponibilidad léxica del grupo" corresponde al subconjunto de palabras de mayor utilización en dicho proceso comunicativo (Michea, 1953).

Los estudios de disponibilidad léxica comenzaron en la década de los cincuenta por la Unesco, buscando una forma de representar un vocabulario común básico de la lengua francesa para facilitar el aprendizaje de dicha lengua a los habitantes no nativos de Francia (López, 1993). Para obtener el léxico disponible de un grupo de personas se realiza un tipo de prueba de fluencia semántica denominada "Prueba de disponibilidad léxica", que consiste en indicar a los individuos que escriban en orden las primeras palabras que le vengan a la mente ante un estímulo que representa el contexto y tema (centro de interés), que son enunciados generalmente mediante una palabra, frase u oración simple.

En el ámbito educacional diversos estudios han encontrado relación entre la disponibilidad léxica y la cantidad de años que un estudiante ha permanecido en una institución (Ferreira, Salcedo y Del Valle, 2014; Germany y Cartes, 2000; Salcedo y Del Valle, 2013), encontrándose que a mayor cantidad de años se produce una mayor cantidad de palabras por grupo. Así, el orden de las palabras producidas puede mostrar diferencias a causa del nivel de escolaridad de los alumnos y ciudad de origen (Echeverría, Vargas, Urzua y Ferreira, 2008; Salcedo y Del Valle, 2013). Adicionalmente, algunos enfoques basados en técnicas de reconocimiento de patrones muestran que es posible representar matemáticamente los lexicones para predecir el rendimiento académico de los alumnos en relación con los rangos de sus calificaciones (Rojas, Zambrano y Salcedo, 2016).

En este mismo sentido, la evidencia sobre la relación entre el lexicón de un estudiante y el conocimiento que éste posee sobre un tema de aprendizaje (representado por un centro de interés), permite hacer una analogía entre el concepto de asimetría de conocimientos descrito anteriormente y la asimetría de disponibilidad léxica. En consecuencia, la asimetría de disponibilidad léxica entre los individuos tendría influencia en el nivel de interacción de colaboración. De esta forma, seleccionar a los integrantes de los grupos colaborativos sobre la base de la asimetría de disponibilidad léxica entre sus miembros permitiría controlar en forma indirecta el nivel de interacción colaborativa.

Visto lo anterior, y con el objetivo de seleccionar los miembros de un grupo colaborativo según su asimetría de disponibilidad léxica, es preciso determinar una forma de medir tal asimetría y definir un criterio de conformación de grupo que utilice dicha medición para seleccionar a los integrantes. Por un lado, medir la asimetría de la disponibilidad léxica entre estudiantes equivale a cuantificar la diferencia entre las listas de vocablos más disponibles de cada uno, las que llamaremos "lexicones disponibles" o simplemente "lexicón" con objeto de facilitar la presentación de resultados. Para esto, algunos enfoques de análisis de disponibilidad léxica, como el presentado en Rojas et al. (2017), permiten determinar distancias cuantitativas entre los lexicones de los estudiantes y otorgarle una interpretación numérica a esta asimetría.

Por otro lado, conformar los grupos de trabajo colaborativos de forma intencionada mediante características cuantificables es un problema matemático/algorítmico que ya ha sido abordado por diversas investigaciones (Burlak y Steinmetz, 2014; Korf y Richard, 1998; Konert, Pinninghoff, Contreras y Salcedo, 2016). En estos trabajos se muestra cómo se puede utilizar cualquier característica cuantificable de los alumnos para maximizar o minimizar la heterogeneidad de los miembros en cada grupo y homogeneizar los grupos entre sí, con tal de que exista un balance entre las características medidas de cada agrupación y no producir configuraciones grupales que den ventaja a unos sobre otros. 
Teniendo en cuenta la dificultad de conformar grupos automáticamente, y sobre la base de la utilidad que un método de estas características podría suponer al ser utilizado en el aula, en las siguientes secciones proponemos un método de conformación automática de grupos colaborativos que utiliza la prueba de disponibilidad léxica como criterio. El apartado metodológico muestra los fundamentos para el diseño y prueba del método, luego se presentan los resultados y el análisis de un estudio de caso donde este método es aplicado, para finalizar con la discusión de los aportes y alcances realizados por esta investigación.

\section{Método}

Este trabajo propone un método de conformación de grupos colaborativos a través del criterio de asimetría de disponibilidad léxica descrito en apartados anteriores. Los fundamentos se basan en las teorías y aplicaciones de los estudios de disponibilidad léxica, con tal de influir en los niveles de interacción colaborativa entre los miembros del grupo.

En este contexto, se ha realizado un estudio descriptivo-exploratorio de dos etapas sobre la relación entre la disponibilidad léxica de los alumnos y el trabajo colaborativo. Para este diseño, en una primera etapa, se procedió a realizar la definición del método de conformación de grupos propuesto mediante el análisis de disponibilidad léxica sobre 394 alumnos de primero a cuarto año de la carrera de Pedagogía en Matemática y Computación de la Universidad de Concepción (Chile). Lo anterior se realizó con el objetivo de determinar los elementos cuantificables que el análisis de disponibilidad léxica ofrece para fundamentar y establecer una posible relación entre la disponibilidad léxica y las características de los alumnos, como sexo, rendimiento y permanencia en la universidad.

En la segunda etapa, con el fin de cuantificar la influencia del método propuesto (conformación de grupos basado en disponibilidad léxica), se procedió a diseñar una actividad para la aplicación de éste en un estudio de caso con 18 estudiantes de primer año de la carrera de Educación Diferencial de la Universidad de Concepción. Esta muestra generó, en una única sesión, 72 registros compuestos por 5 indicadores determinados para la actividad.

La actividad consistió en la lectura de un documento breve que presentaba conceptos generales e históricos de la programación, principalmente con fines informativos y motivacionales. Luego, tras la conformación de parejas realizada por el software que implementa el método propuesto, se procedió a la actividad colaborativa que corresponde a un conjunto de ejercicios de resolución de problemas de programación de corta duración. El tutorial de programación es de acceso público y está enmarcado en la iniciativa mundial "La hora del código", la cual tiene su versión en Chile y cuenta con el apoyo gubernamental. ${ }^{1}$ El tutorial estuvo compuesto por 20 actividades de programación denominadas "Niveles", de las cuales 2 fueron utilizadas como demostración (desarrolladas por el profesor) y las otras 9 fueron realizadas por los grupos colaborativos, utilizando un solo computador a la vez. Durante el desarrollo de la actividad un software instalado en el computador registró las siguientes interacciones de los integrantes del grupo con el tutorial:

Tamaño de respuesta: un mismo problema tiene múltiples soluciones, constituyendo el número de elementos utilizados para resolverlos su indicador de calidad de respuesta (tamaños más pequeños indican mayor calidad).

\% Soluciones óptimas: existe para cada Nivel una cantidad mínima de elementos para resolver el problema. Cuando los alumnos proveen una solución con la cantidad idónea se le considera como una solución óptima. Por lo tanto, este indicador mide el porcentaje de soluciones óptimas realizadas por los estudiantes en las 9 actividades colaborativas.

Número de reintentos: este indicador mide la cantidad de intentos realizados por los alumnos para solucionar un Nivel. No considera el intento exitoso (sea óptimo o no).

\footnotetext{
${ }^{1}$ http://www.horadelcodigo.cl
} 
Choques: cada Nivel tiene elementos en pantalla que, de ser utilizada una programación incorrecta, puede provocar la colisión de ciertos elementos que conducen a una solución fallida. Este indicador mide la cantidad de veces en que no se llegó a la solución por colisionar elementos.

Tiempo: este indicador mide el tiempo en segundos que demora un grupo en resolver un Nivel.

\section{Método de conformación de grupos mediante disponibilidad léxica}

Este trabajo utiliza la distancia entre lexicones definida en Rojas et al. (2017) para hacer las comparaciones; dicha medida, basada en la Distancia de Levensthein (Yujian y Bo, 2007), permite diferenciar numéricamente dos lexicones de tal forma que, a mayor valor mayor es la diferencia entre los lexicones.

Para verificar la factibilidad de utilizar la disponibilidad léxica como criterio de conformación de grupos se realizó un estudio exploratorio sobre el enfoque de distancia propuesto en Rojas et al. (2017) para establecer relaciones grupales.

La muestra utilizada para determinar esta factibilidad corresponde a los datos recolectados mediante el instrumento de Test de Disponibilidad léxica empleado por Valencia y Echeverría (1999), con un tiempo límite de respuesta de dos minutos por cada centro de interés. Se aplicó esta prueba a los alumnos de la carrera de Pedagogía en Matemática y Computación de la Universidad de Concepción, consistente en alumnos de cuatro niveles (de primero a cuarto año). Con esta prueba se obtuvieron 394 lexicones, de cinco centros de interés definidos a partir de la agrupación por temática de los 21 estándares disciplinares que determina el Ministerio de Educación de Chile para la enseñanza de las matemáticas en educación media (Mineduc, 2012); estos son: Sistemas Numéricos y Álgebra (NUM), Cálculo (CALC), Estructuras Algebraicas (ESTR), Geometría (GEOM), y Datos y Azar (AZAR).

Del análisis efectuado no se encontró relación entre el género y la distancia entre lexicones en un nivel significativo para la muestra completa. Sin embargo, realizando una reagrupación de esta distancia, definiendo dos grupos formados por las distancias menores a la media (BAJA) y otro grupo con distancias mayores o iguales a la media (ALTA), se encontró, mediante una prueba Chi-Cuadrado, que la distancia de los lexicones al grupo (ALTA o BAJA) difería significativamente en función del género $x^{2}(1, N=79)=7.9, p<$ 0.005. Esto fue observado sólo para el centro de interés GEOM.

En lo referente a la cantidad de vocablos por respuesta y su relación con la distancia de la disponibilidad léxica, se encontraron correlaciones significativas entre éste y la Distancia de Levenshtein al centro de interés Estructuras, específicamente para los alumnos con uno a tres años de permanencia en la universidad con una significación de г (25) = 0.55, $p<0.004 ;\ulcorner(11)=0.87, p<0.001 ;$ г $(20)=0.57, p<0.008$ respectivamente.

En este mismo contexto, se debe indicar que ya existía evidencia entre las diferencias de disponibilidad léxica producidas por los años de permanencia en la universidad (Ferreira et al., 2014; Salcedo y Del Valle, 2013). Además, se ha encontrado relación entre la cohesión de los lexicones y el desempeño en pruebas de lectura-escritura (Cepeda et al., 2017). Igualmente, en Rojas et al. (2016) se aporta evidencia de la relación entre el léxico disponible y el rendimiento académico, donde a través de técnicas de reconocimiento de patrones fue posible obtener hasta un 83\% de precisión en la predicción de la nota de un alumno.

En consecuencia, sobre la base del marco teórico de la investigación y lo presentado en este apartado, se puede deducir que existe la posibilidad de distinguir numéricamente el lexicón de los estudiantes de distintos niveles, que a su vez provienen de diferentes centros de interés y sobre los cuales puede haber un efecto derivado del género.

Según las consideraciones presentadas, para conformar los grupos se utilizó un enfoque exhaustivo, buscando obtener lo óptimo de una configuración mediante la función F presentada en la ecuación 1. De 
esta forma, para encontrar el valor mínimo de F se debe calcular la heterogeneidad de cada grupo (HET) y la homogeneidad entre grupos (HOM) definidos en las ecuaciones 2 y 3 , respectivamente.

$$
\begin{gathered}
H E T_{G}=\sum_{i, j \in G} D(i, j) \\
H O M=\sum_{n, m \in T \wedge}\left|H E T_{n}-H E T_{m}\right| \\
F=\frac{1}{H O M} \sum_{g \in T} H E T_{g}
\end{gathered}
$$

En primer lugar, la ecuación 1 define a HET, que mide la heterogeneidad de una conformación específica de un grupo G, sumando la distancia entre lexicones $D$ de todos sus integrantes $i$ y $j$. Aquí, la distancia entre lexicones D corresponde a la distancia definida por Rojas et al. (2017) para comparar la disponibilidad léxica entre dos individuos. Esta distancia está basada en la distancia de Levenshtein y su principal característica es ser una propuesta algorítmica que no utiliza la frecuencia de las palabras derivadas del grupo para su cálculo.

En segundo lugar, la ecuación 2 (HOM) se define como la suma del valor absoluto de las diferencias de todas las medidas de heterogeneidad de cada grupo; es decir, mide cuán disímiles son en total todos los grupos que se han conformado, siendo $n$ y $m$ dos grupos que pertenecen al grupo total de alumnos (T). En último lugar, la ecuación 3 muestra la definición de (F) como la razón entre la suma de todas las heterogeneidades de todos los grupos (G) que pertenecen al total de alumnos ( $T$ ) versus la medida de homogeneidad de todos los grupos (HOM).

A pesar de que para buscar un valor óptimo de $\mathrm{F}$ existen varios enfoques de solución (Korf y Richard, 1998; Konert et al., 2014; Pinninghoff et al., 2016), en la presente investigación la cantidad de alumnos utilizados en el estudio de caso es lo suficientemente pequeño como para utilizar un enfoque de solución exhaustivo, que consiste en probar todas las combinaciones posibles y luego evaluar F. Esto trae por consecuencia que la solución entregada será la óptima, teniendo la seguridad de que, bajo el criterio propuesto para seleccionar a los integrantes, no existirá una conformación mejor, lo que no podría ser asegurado por enfoques basados en soluciones aproximadas.

\section{Resultados del estudio de caso}

La muestra estuvo integrada por 18 estudiantes de primer año de la carrera de Educación Diferencial de la Universidad de Concepción (Chile). La elección de un género homogéneo fue para controlar esta variable que puede tener efecto en la cuantificación de la disponibilidad léxica (según análisis presentado en el apartado anterior). Además, se eligió realizar el estudio de caso en una carrera que no tuviese relación con el tutorial a utilizar, ya que el grupo empleado para buscar relaciones y establecer el modelo matemático correspondía a una carrera que tiene computación como parte de su currículo (Pedagogía en Matemática y Computación). De esta forma, las estudiantes formaron 4 grupos mediante el método de conformación propuesto (AUTO), y 5 en forma aleatoria (AZAR). Cada pareja, independiente de su tipo de conformación, desarrolló 9 actividades. Las interacciones con el software resultaron en 72 registros, compuestos por los indicadores presentados en la sección anterior. El análisis de disponibilidad léxica y los resultados de las pruebas se presentan a continuación. 


\subsection{Estadígrafos de disponibilidad léxica}

Los índices de disponibilidad léxica grupales obtenidos incluyen 69 palabras diferentes (NPD), lo que influye en un índice de cohesión (Ic) con valor 0.1060, indicando una alta producción de palabras distintas $X R=7.31 \pm 3.33$. La tabla I muestra el índice de disponibilidad léxica (IDL) para los 15 vocablos más disponibles para el total de alumnos (TOTAL), y para los grupos conformados al azar (AZAR) y por el método propuesto (AUTO). Para una correcta presentación de los vocablos en tablas y gráficos se han omitido los acentos intencionalmente en todos los resultados presentados.

Tabla I. IDL para los 15 vocablos de mayor disponibilidad para el total de alumnos (TOTAL), los grupos conformados al azar (AZAR) y los conformados automáticamente (AUTO)

\begin{tabular}{c|c|c|c|c|c|c}
\cline { 2 - 7 } \multicolumn{2}{c|}{ TOTAL } & \multicolumn{2}{c}{ AZAR } & \multicolumn{2}{c}{ AUTO } \\
\hline Posición & Vocablo & IDL & Vocablo & IDL & Vocablo & IDL \\
\hline 1 & programa & 0.458 & programa & 0.399 & programa & 0.555 \\
2 & computador & 0.334 & computador & 0.381 & organizacion & 0.393 \\
3 & sistema & 0.309 & sistema & 0.294 & computacion & 0.333 \\
4 & organizacion & 0.225 & internet & 0.292 & sistema & 0.333 \\
5 & internet & 0.182 & software & 0.190 & plan & 0.317 \\
6 & software & 0.141 & alinear & 0.147 & computador & 0.257 \\
7 & tecnologia & 0.137 & ejecutar & 0.139 & tiempo & 0.244 \\
8 & tiempo & 0.125 & juegos & 0.133 & software & 0.243 \\
9 & computacion & 0.119 & enlace & 0.132 & canal & 0.178 \\
10 & plan & 0.119 & ordenar & 0.132 & tecnologia & 0.162 \\
11 & planificar & 0.108 & tecnologia & 0.128 & matematica & 0.150 \\
12 & planificacion & 0.104 & organizacion & 0.125 & planificar & 0.150 \\
13 & instalacion & 0.102 & adecuacion & 0.112 & estructura & 0.143 \\
14 & alinear & 0.101 & union & 0.100 & creacion & 0.135 \\
15 & ejecutar & 0.092 & Planificar & 0.100 & instalacion & 0.135 \\
\hline
\end{tabular}

Dentro de los enfoques alternativos de representación de la disponibilidad léxica, la figura 1 muestra un grafo que representa la red semántica de relaciones entre los vocablos más disponibles. En esta figura, los nodos representan los vocablos y su tamaño la disponibilidad de dicho vocablo (IDL). La arista entre los nodos muestra la relación entre los nodos y está dada por la distancia entre los vocablos obtenida mediante la representación vectorial presentada por Rojas et al. (2016). De este modo, una arista más gruesa equivale a una mayor cercanía de los vocablos y una mayor coocurrencia en los distintos lexicones. Por ejemplo, el vocablo programa tiene el IDL más alto, mientras que la relación entre plan y tiempo es la más cercana.

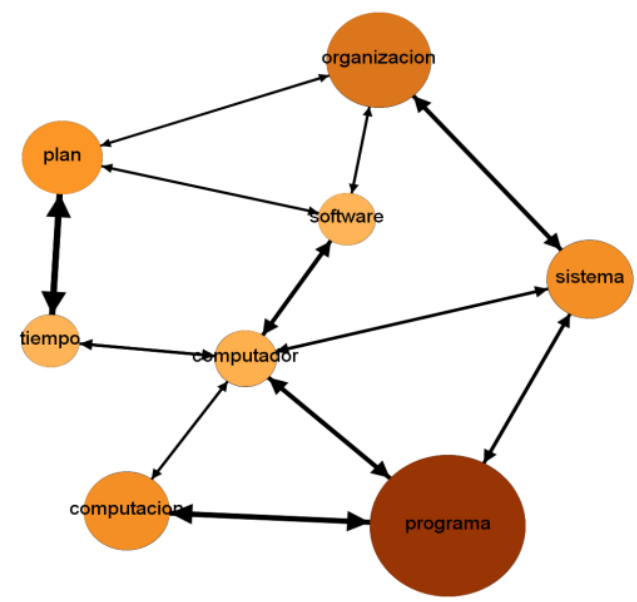

Figura 1. Grafo de los vocablos más disponibles. Se presentan los vocablos con IDL > 0.229 


\subsection{Indicadores de interacción con el tutorial}

La tabla II presenta los estadísticos descriptivos para la interacción del tutorial con los grupos conformados automáticamente (AUTO) y al azar (AZAR). Estos corresponden a la descripción de todos los niveles desarrollados por cada grupo. Como se aprecia, los grupos AZAR tienen una distancia léxica con menor variación que la de AUTO.

Tabla II. Estadísticos descriptivos para la interacción de los usuarios con el tutorial

\begin{tabular}{c|c|c|c|c|c|c|c}
\hline $\begin{array}{c}\text { Tipo } \\
\text { grupo }\end{array}$ & $\begin{array}{c}\text { Distancia } \\
\text { léxica }\end{array}$ & $\begin{array}{c}\text { Tamaño } \\
\text { respuesta }\end{array}$ & $\begin{array}{c}\% \text { Soluciones } \\
\text { óptimas }\end{array}$ & $\begin{array}{c}\text { Número de } \\
\text { reintentos }\end{array}$ & Fallos & Choques & Tiempo \\
\hline AZAR & $7.25 \pm 3.3$ & $8.15 \pm 5.09$ & $61 \% \pm 2.44 \%$ & $1.34 \pm 3.78$ & $0.07 \pm 0.07$ & $0.85 \pm 1.98$ & $138.98 \pm 113,96$ \\
\hline AUTO & $7.33 \pm 1.53$ & $6.32 \pm 2.28$ & $64 \% \pm 2.42 \%$ & $0.82 \pm 2.72$ & $0.14 \pm 0.12$ & $1.32 \pm 7.37$ & $177.55 \pm 176.45$ \\
\hline
\end{tabular}

La tabla III muestra una visión pormenorizada de los detalles de interacción del software con los usuarios. Como se observa, el tiempo de la primera actividad es mayor a los otros. Además, los tiempos para los grupos AUTO son en general mayores a los de AZAR.

Tabla III. Estadísticos descriptivos para la interacción del usuario por cada nivel

\begin{tabular}{|c|c|c|c|c|c|c|}
\hline Nivel & $\begin{array}{l}\text { Tipo } \\
\text { grupo }\end{array}$ & $\begin{array}{l}\text { Tamaño } \\
\text { respuesta }\end{array}$ & $\begin{array}{c}\text { \% Soluciones } \\
\text { óptimas }\end{array}$ & $\begin{array}{l}\text { Número de } \\
\text { reintentos }\end{array}$ & Choques & Tiempo \\
\hline \multirow[t]{2}{*}{3} & AZAR & $5.00 \pm 0.00$ & $100 \pm 0.0$ & $2.20 \pm 1.30$ & $0.40 \pm 0.89$ & $267.20 \pm 251.64$ \\
\hline & AUTO & $5.00 \pm 0.00$ & $100 \pm 0.0$ & $0.67 \pm 0.58$ & $0.00 \pm 0.00$ & $390.33 \pm 311.92$ \\
\hline \multirow[t]{2}{*}{4} & AZAR & $5.60 \pm 0.55$ & $100 \pm 0.0$ & $0.00 \pm 0.00$ & $0.60 \pm 0.55$ & $60.40 \pm 33.31$ \\
\hline & AUTO & $5.67 \pm 0.58$ & $100 \pm 0.0$ & $0.00 \pm 0.00$ & $0.00 \pm 0.00$ & $30.67 \pm 3.79$ \\
\hline \multirow[t]{2}{*}{5} & AZAR & $9.20 \pm 0.45$ & $80 \pm 45$ & $0.00 \pm 0.00$ & $0.40 \pm 0.89$ & $60.80 \pm 26.03$ \\
\hline & AUTO & $9.00 \pm 0.00$ & $100 \pm 0.0$ & $0.33 \pm 0.58$ & $1.33 \pm 1.15$ & $135.00 \pm 59.19$ \\
\hline \multirow[t]{2}{*}{6} & AZAR & $8.20 \pm 1.79$ & $60 \pm 55$ & $0.20 \pm 0.45$ & $0.00 \pm 0.00$ & $67.60 \pm 39.04$ \\
\hline & AUTO & $9.00 \pm 0.00$ & $00 \pm 00$ & $3.33 \pm 3.21$ & $0.00 \pm 0.00$ & $150.67 \pm 73.91$ \\
\hline \multirow[t]{2}{*}{7} & AZAR & $5.00 \pm 4.12$ & $60 \pm 55$ & $3.00 \pm 2.00$ & $0.00 \pm 0.00$ & $173.40 \pm 54.28$ \\
\hline & AUTO & $2.00 \pm 0.00$ & $100 \pm 00$ & $0.33 \pm 0.58$ & $0.00 \pm 0.00$ & $139.33 \pm 166.33$ \\
\hline \multirow[t]{2}{*}{8} & AZAR & $9.00 \pm 5.48$ & $60 \pm 55$ & $1.60 \pm 3.05$ & $0.80 \pm 1.30$ & $137.40 \pm 107.27$ \\
\hline & AUTO & $5.00 \pm 0.00$ & $100 \pm 00$ & $0.00 \pm 0.00$ & $0.00 \pm 0.00$ & $63.33 \pm 43.36$ \\
\hline \multirow[t]{2}{*}{9} & AZAR & $12.60 \pm 8.29$ & $20 \pm 45$ & $1.20 \pm 1.79$ & $0.80 \pm 0.45$ & $146.40 \pm 99.12$ \\
\hline & AUTO & $8.67 \pm 1.15$ & $0 \pm 00$ & $0.00 \pm 0.00$ & $0.67 \pm 0.58$ & $76.00 \pm 13.53$ \\
\hline \multirow[t]{2}{*}{10} & AZAR & $6.80 \pm 2.17$ & $20 \pm 45$ & $1.20 \pm 1.30$ & $1.80 \pm 2.95$ & $200.40 \pm 176.60$ \\
\hline & AUTO & $6.67 \pm 2.31$ & $33 \pm 58$ & $0.33 \pm 0.58$ & $1.00 \pm 1.00$ & $143.33 \pm 14.01$ \\
\hline \multirow[t]{2}{*}{11} & AZAR & $11.00 \pm 8.22$ & $60 \pm 55$ & $2.00 \pm 2.83$ & $2.40 \pm 1.14$ & $186.40 \pm 142.21$ \\
\hline & AUTO & $5.33 \pm 0.58$ & $67 \pm 58$ & $2.00 \pm 1.73$ & $7.33 \pm 3.21$ & $397.33 \pm 105.19$ \\
\hline
\end{tabular}

Para buscar diferencias significativas entre los resultados, se realizaron pruebas estadísticas inferenciales no paramétricas, U de Mann-Whitney para muestras independientes. Así, definiendo para cada nivel a la variable independiente "Tipo de Grupo" (AZAR y AUTO), y como variable dependiente a cada indicador, se obtuvieron diferencias significativas para el Nivel 6 y la variable "Número de intentos" $(U=14.5, p<0.036)$, y para el Nivel 11, la variable "Choques" $(U=15, p<0.036)$.

\section{Discusión}

Con los resultados obtenidos se obtiene una perspectiva amplia de la disponibilidad léxica de los participantes en el estudio de caso. Según la tabla I, los vocablos más disponibles están altamente 
relacionados con el concepto de computación (en el sentido de tecnología), algo esperable debido al centro de interés "programación" presentado. A pesar de que en esta tabla los vocablos relacionados al concepto de programación como una actividad o acción tienen una baja disponibilidad, se puede apreciar en la figura 1 que el vocablo organización está fuertemente relacionado con el vocablo sistema. Esto implica que aun cuando son menos disponibles los vocablos referidos a la programación como una actividad, estos son producidos por los estudiantes frecuentemente en conjunto con el vocablo sistema.

De lo anterior se puede deducir que los alumnos relacionaron el concepto "programación" con la "computación" como "tecnología", aunque también asociaron este centro de interés con vocablos referentes a "planificar" y "organizar tareas", por lo que los objetivos del tutorial de programación no serían ajenos a este hecho, debido a que precisamente presenta actividades de organización y planificación de tareas en un sistema computacional.

En lo referente a los resultados de interacción con el sistema tutorial, en ambos grupos se puede indicar que lo más relevante corresponde a los indicadores de las variables Tamaño de respuesta y Número de intentos. En este sentido, en la tabla III se muestra que a partir del Nivel 7 el tamaño de las respuestas de los grupos AUTO es menor que el de las del grupo AZAR. Al respecto, desde el Nivel 7 en delante, el tutorial presenta otro tipo de estructuras de programación posibles de utilizar para la solución, donde existe la posibilidad de utilizar elementos simples de programación (lo que produce respuestas más largas) versus estructuras más complejas en anidación (lo que origina respuestas más cortas). La diferencia en el indicador Tamaño de respuesta puede ser producto de que los alumnos en estos grupos utilizaron los elementos de programación en forma no mecánica, reflejando una mayor apertura a utilizar elementos nuevos de programación, en lugar de reutilizar soluciones básicas aprendidas en niveles anteriores. Por el contrario, el grupo AZAR presenta soluciones largas sobre la base de la repetición manual de elementos básicos y, por consiguiente, una mayor cantidad de reintentos y tiempo de respuesta desde el nivel 7 al 10.

Desde el punto de vista de la disponibilidad léxica y su relación con los resultados, además del posible efecto evidenciado en soluciones más pequeñas, es posible que la mayor heterogeneidad de sus integrantes pueda provocar una asimetría. Y que, dada una actividad desconocida por parte de los integrantes, estos tiendan a interactuar con tal de resolver el problema presentando distintas perspectivas de solución y seleccionando la más acorde. Provocando que las soluciones sean de menor tamaño y los reintentos también sean menores en los niveles 7 a 10. Esto puede suponer que los integrantes de los grupos AUTO tienen, desde el punto de vista de la disponibilidad léxica, un nivel mayor de asociación de vocablos relacionados al concepto de planificación, lo que puede verse en la tabla I, donde precisamente los vocablos como organización tienen una disponibilidad más alta en este último tipo de grupo.

A pesar de lo anterior, no es posible medir adquisición de habilidades o conocimientos con el diseño propuesto (aunque sí el tipo de solución y sus intentos), por lo que no se puede establecer una relación causal de la asimetría de lexicones controlada por la conformación de grupos sobre el nivel de aprendizaje. Además, otros elementos de análisis, como el efecto que esta asimetría puede provocar en la comunicación de los integrantes, no han sido abordados, ya que a pesar de que esta asimetría es controlada por la homogeneidad buscada entre los grupos, una asimetría demasiado alta podría provocar problemas de comunicación que serían contraproducentes con el proceso de colaboración.

\section{Conclusiones}

Sobre la definición del método de conformación de grupos propuesto y su fundamentación, es posible concluir que fue necesaria la utilización de distintos enfoques de cuantificación de la disponibilidad léxica para poder determinar características de ésta que permitan establecer su relación con la interacción de colaboración, principalmente una distancia algorítmica y una función de heterogeneidad intragrupo y homogeneidad entre grupos. Los resultados muestran evidencias de que, comparativamente, la asimetría de lexicones, controlada por la conformación automática de grupos, puede afectar el tipo de solución presentada por los grupos en una tarea de colaboración. 
Es posible igualmente concluir que, aunque no se puede, mediante el diseño propuesto, establecer una relación causal entre la disponibilidad léxica del grupo y los aprendizajes logrados con el tutorial, se muestra evidencia sobre la diferencia significativa de los resultados que probablemente sea producto de la asimetría de disponibilidad de sus participantes.

En el mismo contexto, el método propuesto permite generar mediante una prueba de corta duración (dos minutos aproximadamente) grupos óptimos según la disponibilidad léxica de sus integrantes, conformando grupos que internamente son heterogéneos, pero que entre los distintos grupos son homogéneos. En este sentido, cabe resaltar que debido a que la disponibilidad léxica es contextual y permeable a las condiciones del momento y estado de los individuos, permite establecer los grupos en el contexto inmediato de sus integrantes y difiere de otros métodos de conformación basados en test o pruebas psicométricas que generalmente son de duración más larga y que son medidas una sola vez para cada individuo.

Para finalizar, se puede indicar que, a partir del enfoque de conformación de grupos de colaboración propuesto, nuevas posibilidades de investigación se pueden abrir para la utilización de las pruebas de disponibilidad léxica en el aula, al utilizarla con fines pedagógicos y estudiar su relación no sólo con los niveles de interacción sino con el logro de aprendizajes.

\section{Referencias}

Álvarez, C. (2010). La relación entre lenguaje y pensamiento de Vigotsky en el desarrollo de la psicolingüística moderna. Revista de Lingüística Teórica y Aplicada, 48(2), 13-32.

Arias-Gundín, O., Fidalgo, R. y García, J. (2008). El desarrollo de las competencias transversales en magisterio mediante el aprendizaje basado en problemas y el método de caso. Revista de Investigación Educativa, 26(2), 431-444. Recuperado de https://revistas.um.es/rie/article/view/94011

Ariza A. y Oliva S. (2000). Las nuevas tecnologías de la información y la comunicación y una propuesta para el trabajo colaborativo. Documento presentado en el V Congreso Iberoamericano de Informática Educativa, Chile.

Carrió, M. L. (2007). Ventajas del uso de la tecnología en el aprendizaje colaborativo. Revista Iberoamericana de Educación, 41(4). doi:10.35362/rie4142447

Cepeda, M., Cárdenas, A., Carrasco, M., Castillo, N., Flores, J., González, C. y Oróstica, M. (2017). Relación entre disponibilidad léxica y comprensión lectora, en un contexto de educación técnico profesional rural. Revista Sophia Austral, 1(1), 81-93. Recuperado de http://www.sophiaaustral.cl/index.php/shopiaaustral/article/view/51/46

Coll, C. y Solé, I. (1990). La interacción profesor/alumno en el proceso de enseñanza y aprendizaje. Madrid: Alianza.

Collazos, C. y Mendoza, J. (2006). Cómo aprovechar el "aprendizaje colaborativo" en el aula. Educación y Educadores, 9(2), 61-76.

Del Valle, M., Salcedo, P. y Ferreira, A. (2016). Analyzing the availability of lexicon in mathematics education using no traditional technological resources. Journal of Supply Chain Management, 5(2), 144149. Recuperado de https://ojs.excelingtech.co.uk/index.php/IJSCM/article/view/1177

Echeverría, M., Vargas, R., Urzua, P. y Ferreira, R. (2008). DispoGrafo: una nueva herramienta computacional para el análisis de relaciones semánticas en el léxico disponible. Revista de Lingüistica Teórica y Aplicada, 46, 81-91. doi:10.4067/S0718-48832008000100005 
Ferreira, A., Salcedo, P. y del Valle, M. (2014). Estudio de disponibilidad léxica en el ámbito de las matemáticas. Estudios filológicos, 54, 69-84.

Galindo, L. y Arango, M. (2009). Estrategia didáctica: la mediación en el aprendizaje colaborativo en la educación médica. latreia, 22(3), 284-291. Recuperado de https://aprendeenlinea.udea.edu.co/revistas/index.php/iatreia/article/view/8422

Germany, P. y Cartes, N. (2000). Léxico disponible en inglés como segunda lengua de instrucción formalizada. Estudios Pedagógicos, 26, 39-50. Recuperado de http://revistas.uach.cl/index.php/estped/article/view/2903

Konert J., Burlak D. y Steinmetz R. (2014). The group formation problem: an algorithmic approach to learning group formation. En C. Rensing et al. (Eds.), Proceedings of the 9th European Conference on Technology Enhanced Learning (\#\#\#ECTEL\#\#\#), no. 9, pp. 221-234. Suiza: Springer.

Korf, R. y Richard, E. (1998). A complete anytime algorithm for number partitioning. Artificial Intelligence, 106(2), 181-203. doi:10.1016/S0004-3702(98)00086-1

Lage, F. J. (2001). Ambiente distribuido aplicado a la formación/capacitación de \#\#\#RR\#\#\#\#\#\#HH\#\#\#: un modelo de aprendizaje cooperativo-colaborativo (Tesis Doctoral). Facultad de Informática, Universidad Nacional de la Plata, Argentina.

Lucero, M. (2003). Entre el trabajo colaborativo y el aprendizaje colaborativo. Revista Ibero Americana de Educación, 33(1). doi:10.35362/rie3312923

Maldonado, M. y Vásquez, M. (2008). Experiencia de desarrollo de un proyecto de aprendizaje colaborativo como estrategia formativa. Educación Médica Superior, 22(1). Recuperada de http://scielo.sld.cu/scielo.php?script=sci serial\&pid=0864-2141\&lnq=es\&nrm=iso

Michea, R. (1953). Mots fréquents et mots disponibles, un aspect nouveau de la statistique du langage [Palabras frecuentes y palabras disponibles, un nuevo aspecto de las estadísticas del lenguaje]. Langues Modernes, 47, 338-344.

Ministerio de Educación de Chile. (2012). Estándares orientadores para carreras de pedagogía en educación media. $\quad$ Recuperado http://portales.mineduc.cl/usuarios/cpeip/File/librosestandaresvale/libromediafinal.pdf

Pérez, M. (2007). El trabajo colaborativo en el aula universitaria. Revista de Educación, 13(23). Recuperado de https://www.redalyc.org/pdf/761/76102314.pdf

Pinninghoff, M., Contreras, R. y Salcedo, P. (2016). Genetic algorithms as a tool for structuring collaborative groups. Natural Computing, 16(2),231-239. doi: 10.1007/s11047-016-9574-1

Rojas, D., Zambrano, C., Salcedo, P. (2016). A proposal for predicting academic achievement throught a vectorial model of lexical availability. Actas de la International Conference of Education, Research and Innovation (\#\#\#ICERI\#\#\#) 2016, pp. 2799-2806.

Rojas, D., Zambrano, C. y Salcedo, P. (2017). Una metodología de comparación jerárquica de lexicones en estudiantes de pedagogía. Formación Universitaria, 10(4), 3-14. doi:10.4067/S0718-50062017000400002

Salas, A. (2001). Implicaciones educativas de la teoría sociocultural de Vigotsky. Revista Educación, 25(2), 59-65. Recuperado de https://www.redalyc.org/pdf/440/44025206.pdf

Salcedo, P. y del Valle, M. (2013). Disponibilidad Léxica Matemática en Estudiantes de Enseñanza Media de Concepción, Chile. Atenas, 1(21). 
Valencia, A. y Echeverría, M. (1999). Disponibilidad léxica en estudiantes chilenos. Santiago de Chile: Ediciones Universidad de Chile-Universidad de Concepción.

Yujian, L., y Bo, L. (2007). A normalized Levenshtein distance metric, \#\#\#IEEE\#\#\# transactions on pattern analysis and machine intelligence, 29(6), 1091-1095. doi: 10.1109/TPAMI.2007.1078

Zañartu, L. M. (2013). Aprendizaje Colaborativo: una nueva forma de Dialogo Interpersonal y en la Red, Contexto Educativo, (28). Recuperado de https://s2.likedoc.org/uploads/STE2017/04/25/WpDwNAObYk/24d828c04d99940c9ad1f043d74f0074.p df 